\title{
Infantile Hemangioma: A Retrospective Study
}

\author{
Eva Lydiawati, Iskandar Zulkarnain \\ Departement of Dermatology and Venereology \\ Faculty of Medicine Universitas Airlangga/Dr. Soetomo General Academic Teaching Hospital \\ Surabaya
}

\begin{abstract}
Background: Infantile Hemangioma $(\mathrm{IH})$ is a benign vascular neoplasm and most commonly found in newborns. IH appears in the first few weeks of life and mostly resolve immediately. Early recognition of symptoms, diagnosis, and therapy has an important role in determining the prognosis of this condition. Purpose: The purpose of this study is to evaluate the clinical features of new IH patients in the Pediatric Dermatology Division of the Dermatology \& Venereology Department outpatient clinic of Dr. Soetomo General Hospital Surabaya in 2008-2017. Methods: This is a retrospective descriptive study from the medical records of new IH patients over a period of 10 years (2008-2017). Results: A total of 84 new IH patients were observed in this study. The data showed that: the most age group was $0-1$ year old $(71.43 \%)$, female $(63.1 \%)$, and no family history of a similar condition (94.05\%). The most common complaint reported was the presence of bumps (59.52\%). Most complaints occurred before the patients turned one year old (89.29\%) and involved the facial region (58.33\%). From the clinical features, the most frequent skin lesions were macules $(35.36 \%)$. In addition, there was a combination of macular and nodular lesions in $28.13 \%$ of patients. The most widely used therapy is laser modality found in $55.45 \%$ of patients. Conclusion: Clinical features of IH patients, in accordance with the theory and treatment choices, dependent on the patients' conditions and clinical manifestations of the disease.
\end{abstract}

Keywords: Infantile hemangioma, benign vascular neoplasm, retrospective study.

Correspondence address: Iskandar Zulkarnain, Department of Dermatology and Venereology, Faculty of Medicine, Universitas Airlangga, Dr. Soetomo General Academic Teaching Hospital, Jl. Mayjen Prof. Dr. Moestopo No. 6-8, 60131, Surabaya, Indonesia. Telephone: +6231-5501609, e-mail: zuljazid@yahoo.com.

\section{INTRODUCTION}

Vascular anomaly is one of the commonly found birthmarks. The classification of vascular anomalies is under debate as there is a large variety of clinical features and nomenclature used. ${ }^{1}$ The International Society for the Study of Vascular Anomalies (ISSVA) divides vascular anomalies into neoplasms and malformations. Neoplasm vascular anomaly is divided into several disorders which one of them is hemangioma. ${ }^{1,2}$ According to the ISSVA classification, hemangiomas are classified into infantile hemangiomas (IH) and congenital hemangiomas. Infantile hemangioma $(\mathrm{IH})$ is the most common type of hemangioma. ${ }^{2,3}$

IH is a benign tumor and is often found in newborns. The prevalence is estimated to be $2-3 \%$ in infants. $^{3-5}$ About $10 \%$ of the case is found in babies aged less than one year, and around $22-30 \%$ of the case is found in babies born with bodyweight $<1,000$ grams. ${ }^{3}$ Some statistical data show that IH is more common in females than males with a prevalence ratio of $3-5: 1 .^{5}$ The condition is also more common in caucasian races. ${ }^{5,6}$ There is no data on hemangioma data in the general Indonesian population.

Most IH cases can be cured immediately. Various treatment options, both topical and systemic, are available depending on the symptoms and severity. The prognosis of $\mathrm{IH}$ is determined by the number of lesions, location, speed of growth and accuracy of diagnosis, and appropriate treatment. ${ }^{7,8}$ Thus, early recognition of symptoms, diagnosis, and therapy play an important role in determining the prognosis of this condition.

\section{METHODS}

This is a retrospective descriptive study of medical records of new $\mathrm{IH}$ patients in the Pediatric Dermatology Division of the Dermatology \& Venereology Department outpatient clinic of Dr. Soetomo General Hospital Surabaya from 2008 to 2017 (10 years). The data were recorded in the form of a total of new patient visits, general descriptions of the patient, including age, sex, clinical data, and IH management. This research has obtained ethical approval from the Ethics Committee of Dr. Soetomo General Hospital Surabaya.

\section{RESULTS}

Between 2008 and 2017, there were $84 \mathrm{IH}$ patients, and they made up $0.69 \%$ of the total patients of the Pediatric Dermatology Division of the 
Dermatology \& Venereology Department outpatient clinic of Dr. Soetomo General Hospital Surabaya. The number is equivalent to $0.04 \%$ of 200,998 patients who visited the Dermatology \& Venereology Department outpatient clinic of Dr. Soetomo General Hospital Surabaya. Detailed information can be observed in Figure 1 below.
Between 2008 and 2017, there were 60 (71.43\%) patients aged less than one year. The youngest patient was one month old, and the oldest was 13 years old. In addition, there were $53(63.1 \%)$ female patients and 31 male patients $(36.9 \%)$. Thus, most patients were aged less than one year old and were female. The age distribution of the sexes can be observed in Table 1 .

The Number of New Cases in 2008-2017

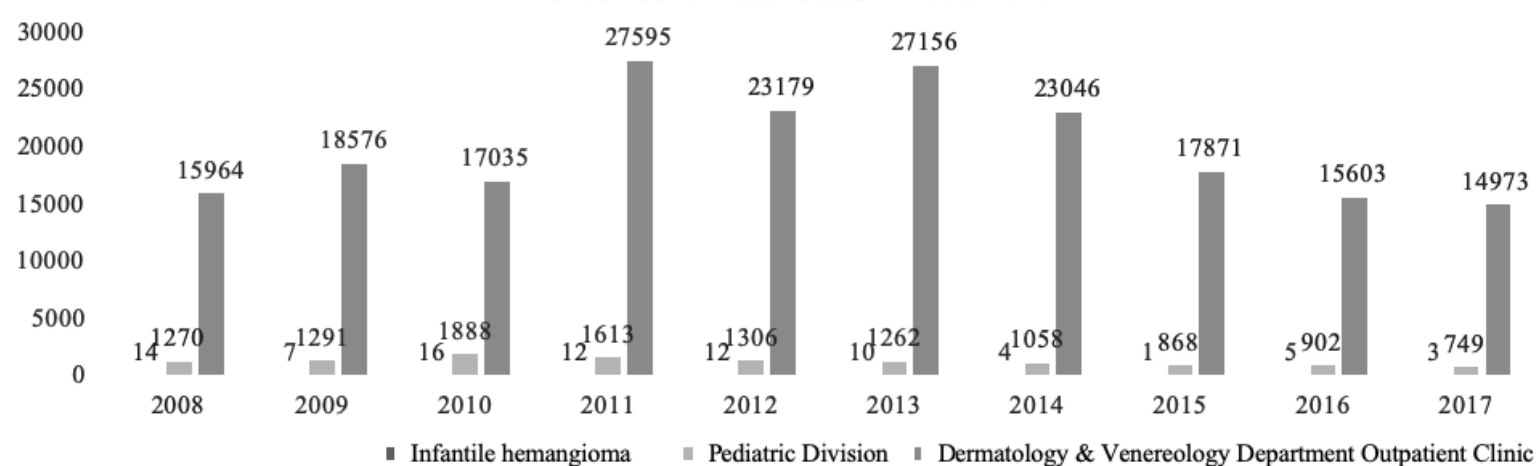

Figure 1. The number of new Infantile Hemangioma patient visits in the Pediatric Dermatology Division of the Dermatology \& Venereology Department outpatient clinic of Dr. Soetomo General Hospital Surabaya from 2008 to 2017.

Table 1. Age and sex-wise distribution of new Infantile Hemangioma patients in the Pediatric Dermatology Division of the Dermatology \& Venereology Department outpatient clinic of Dr. Soetomo General Hospital Surabaya in 2008-2017

\begin{tabular}{|c|c|c|c|}
\hline \multirow{2}{*}{ Age Group (Year) } & \multicolumn{2}{|c|}{ Sex } & Total (\%) \\
\hline & Male & Female & \\
\hline $0-<1$ & 19 & 41 & $60(71.43)$ \\
\hline $1-4$ & 6 & 8 & 14 (16.67) \\
\hline $5-14$ & 6 & 4 & $10(11.90)$ \\
\hline Total & $31(36.9)$ & $53(63.1)$ & $84(100)$ \\
\hline
\end{tabular}

There were two patients $(2.38 \%)$ with infantile hemangioma history running in the family. The majority of the patients, 79 (94.05\%), did not have a family history with $\mathrm{IH}$. A more detailed data on the family history of infantile hemangioma can be found in Figure 2.

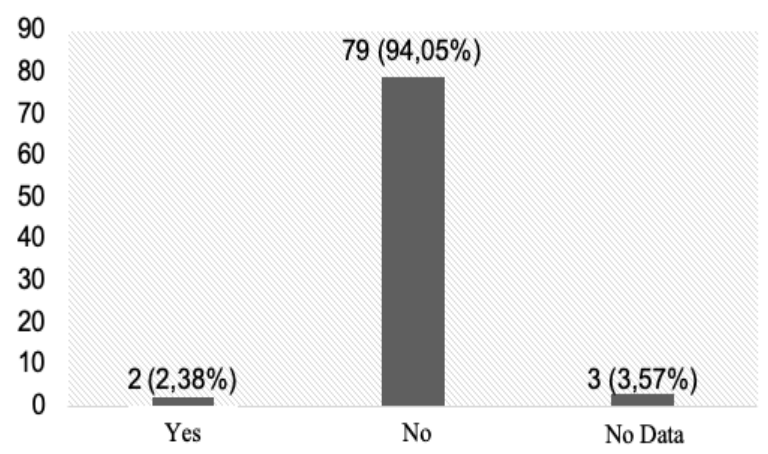

Figure 2. Family history of infantile hemangioma patients in the Pediatric Dermatology Division of the Dermatology \&
Venereology Department outpatient clinic of Dr. Soetomo General Hospital Surabaya from 2008 to 2017.

Most complaints, which were found in 75 patients (89.29\%), occurred before the patients turned one year old. The most common complaint reported was the presence of bumps in 50 patients (59.52), red marks in 33 patients (39.29\%), and followed by sores or blisters in 1 patient (1.19\%) as shown in Figure 3.

The most common IH location was the facial region, with 49 patients $(58.33 \%)$. The other common location of lesions was the superior and inferior extremities with 7 patients $(8.34 \%)$ in each of these regions. In addition, the most common clinical features of the lesion were macules, which were found in 41 patients $(35.36 \%)$. The other less common clinical features of lesions were tumors or nodules, which were found in 33 patients $(28.46 \%)$, and plaques which were found in 20 patients $(17.24 \%)$. 


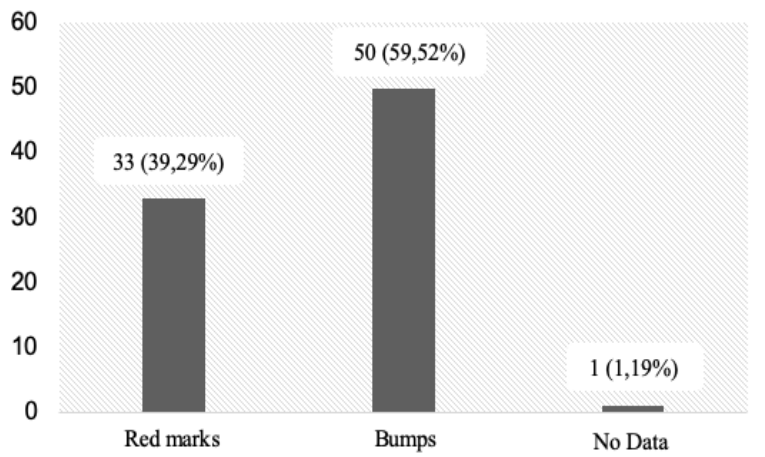

Figure 3. Chief complaints of new infantile hemangioma patients in the Pediatric Dermatology Division of the Dermatology \& Venereology Department outpatient clinic of Dr.

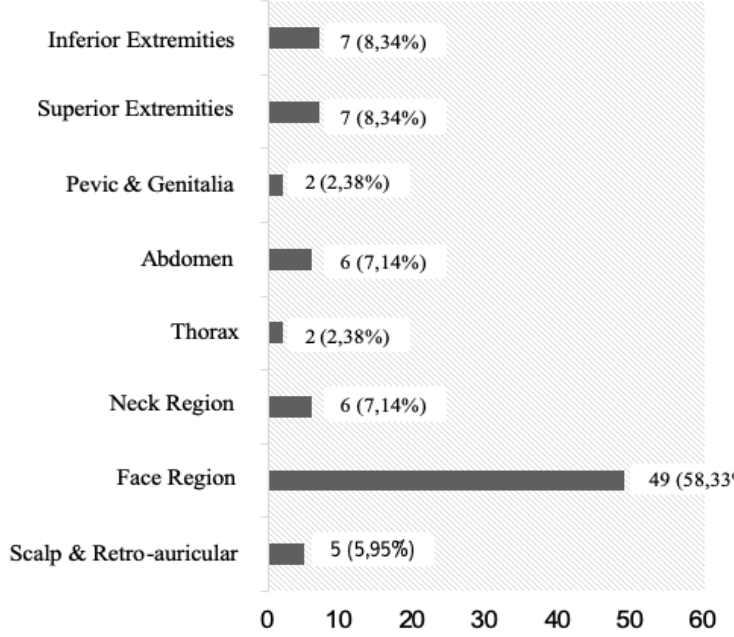

Figure 4. Locations of skin lesions of infantile hemangioma patients in the Pediatric Dermatology Division of the Dermatology \& Venereology Department outpatient clinic of Dr. Soetomo General Hospital Surabaya from 2008 to 2017.
Soetomo General Hospital Surabaya from 2008 to 2017.

The most common combination of IH lesions was macules with tumors or nodules, which were found in 9 patients $(28.13 \%)$. Other quite common combinations were macules with plaques and macules with erosion, which were found in 6 patients, respectively (18.75\%). Thirty-two $\mathrm{IH}$ patients had a combination of skin lesion clinical.

There was no adequate information about the size of the skin lesions. From the available data, 12 patients (14.29\%) had $\geq 50 \mathrm{~mm}^{2}$ skin lesion, and 12 patients (14.29\%) had $\geq 20 \mathrm{~mm}^{2}$ skin lesion.

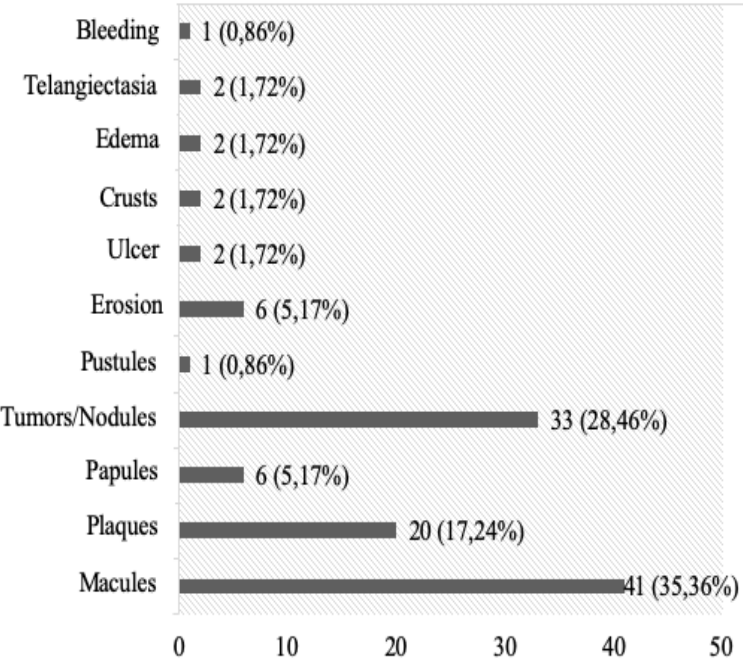

Figure 5. Clinical features of skin lesions of infantile hemangioma patients in the Pediatric Dermatology Division of the Dermatology \& Venereology Department outpatient clinic of Dr. Soetomo General Hospital Surabaya from 2008 to 2017.

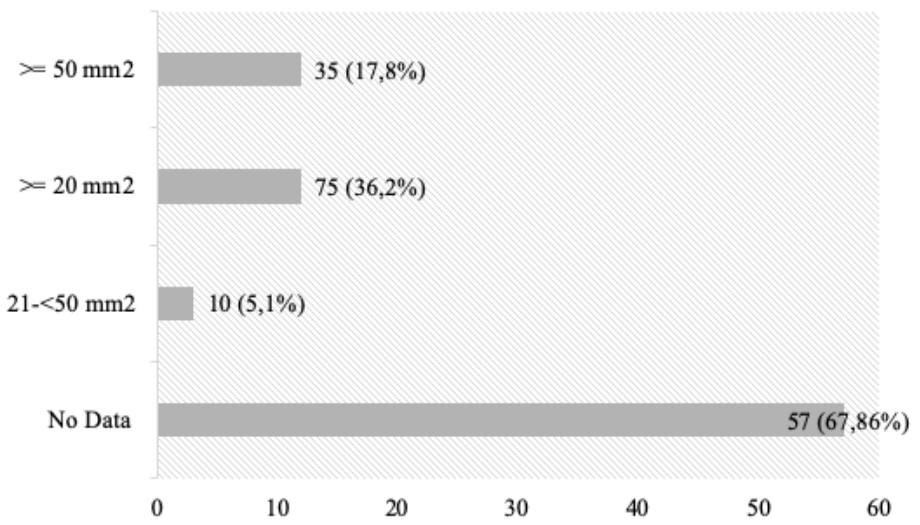

Figure 6. Skin lesions sizes of infantile hemangioma patients in the Pediatric Dermatology Division of the Dermatology \& Venereology Department outpatient clinic of Dr. Soetomo General Hospital Surabaya from 2008 to 2017. 
Laser modality was the most widely used therapy option amounting to 56 patients $(55.45 \%)$. Some patients received more than one therapy. Laser modality and treatment with antibiotics were the most common combination therapy, and it was found in 6 patients $(35.29 \%)$. There were 17 infantile hemangiomas patients who received combination therapy.

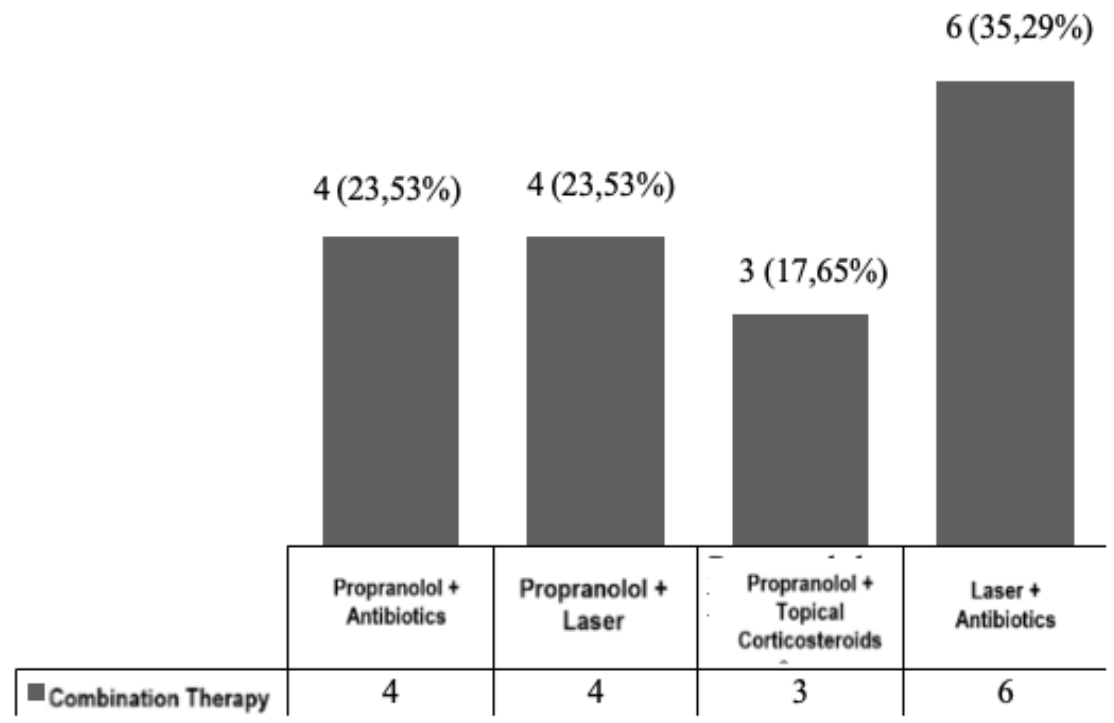

Figure 7. Infantile hemangioma therapy options in the Pediatric Dermatology Division of the Dermatology \& Venereology Department outpatient clinic of Dr. Soetomo General Hospital Surabaya from 2008 to 2017.

\section{DISCUSSION}

A total of $84 \mathrm{IH}$ patients were recorded between 2008 and 2017. From the data, there were $16 \mathrm{IH}$ patients $(19.05 \%)$ in 2010, making 2010 the year with the highest number of IH patients. In 2008, there were $14 \mathrm{IH}$ patients (16.67\%) and each 12 patients (14.29\%) in 2011 and 2012. Those number tended to decrease repeatedly. The number reduction of $\mathrm{IH}$ patients was in line with the total number of patient in other divisions in the Dermatology \& Venereology Department outpatient clinic of Dr. Soetomo General Hospital Surabaya that were also decline in the same period.

The decrement of patient visits was thought to be caused by several factors. One of them was the reluctance of patients to go to the hospital. In this very modern era, people have more accessible health information access through the internet. Abundant information should encourage patients to have an early check-up, but it actually discourages them from visiting the hospital because they prefer other alternative treatments. Furthermore, Indonesia's universal health coverage program (Badan Penyelenggara Jaminan Sosial Kesehatan) requires a multilevel referral system, preventing patients from receiving immediate treatment at Dr. Soetomo General Hospital, which is the largest referral hospital in eastern Indonesia.

The most common age group of IH patients was 0 -1 year old, with 60 patients $(71.43 \%)$. This is in accordance with Leaute-Labreze et al. (2017) research, supporting that infantile hemangiomas are often found in babies aged less than one year old. ${ }^{3}$ This condition occurs in the first few weeks of birth and will develop actively until spontaneous involution at around one year of age. .,9 $^{3}$

Most IH patients were females with 53 patients (63.1\%), and there were 31 male patients $(36.9 \%)$. The previous study that was conducted by George et al. (2014) found that IH is more often found in female than male. ${ }^{2}$ Sex predisposition is also supported by epidemiological data which states that the sex ratio of IH patient is 3-5:1. ${ }^{5}$ This research found that female has a higher prevalence than male, so the sex ratio of $\mathrm{IH}$ patients at Dr. Soetomo between 2008 and 2017 was around 2:1.

Family history of hemangioma could affect the clinical development of IH even though the genetic correlation is still uncertain, while the data from our study demonstrate that most patients did not have IH history. IH has a period of progression and spontaneous regression that varies in each individual and family member unaware of various clinical manifestations of IH. This challenges non-healthcare workers to be aware of these conditions. ${ }^{7,10}$

The most-reported chief complaint of IH patients was the presence of bumps, which was reported by 50 patients $(59.52 \%)$. This is in line with Planasciudad et al. (2017) research, which found that IH can manifest 
as a bluish bump and with indefinite borders. ${ }^{10,11}$ Leaute-Labreze et al. (2017) also stated that the clinical feature of infantile hemangiomas can be in the form of red patches at an early stage and develops into bumps at the later stage. In addition, about $10-15 \%$ of infantile hemangioma cases might lead to complication in the form of scars on the surface of the lesion. ${ }^{3}$

The majority of IH complaints occurred less than one year of age. Most of the onset was found in 75 patients $(89.29 \%)$. IH is a blood vessel tumor that mostly appears in the first year of birth. This condition has a rapid postnatal growth characteristic and is followed by a period of regression during childhood. Hemangioma does not immediately appear after birth but begins to develop in the first year of birth. ${ }^{11,12}$

The most common location of $\mathrm{IH}$ was the facial region, with 49 patients $(58.33 \%)$. The other common locations were the superior and inferior extremities. IH can occur anywhere on the body. ${ }^{12,13}$ Approximately $60 \%$ of $\mathrm{IH}$ are in the craniofacial area, $25 \%$ in the trunk, and $15 \%$ in the extremities. ${ }^{13} \mathrm{IH}$ in the central facial area (including the nose and perioral), the periocular area, and the mandible provide a higher risk of complications compared to other locations. IH on the face is associated with PHACES syndrome (Posterior fossa brain malformations, hemangioma of the face, arterial cerebrovascular anomalies, cardiovascular anomalies, eye anomalies, and sternal defects or supraumbilical raphe). ${ }^{13,14}$ More than one-third of facial hemangioma patients suffer from PHACES if thoroughly inspected. This syndrome causes complications such as seizures, cerebrospinal fluid abnormality, and growth disturbance in the later years. $^{14}$

The most common clinical features of the lesion were macules, which was reported in 41 patients $(35.36 \%)$. The clinical features of these patients were similar with IH lesions development. The lesion may initially be pale due to vasoconstriction or red macules with telangiectasia. After proliferation, the lesion can change in the form of elevated erythematous papules, nodules, or plaque. Profound $\mathrm{IH}$ can extend to the adipose tissue and form bluish tumors. ${ }^{12,15}$

Based on the obtained data, the IH lesion size on 12 patients (14.29\%) was more than or equal to $5 \mathrm{~cm}^{2}$. IHs with a size of less than or equal to $12 \mathrm{~mm}^{2}$ were also found in 12 patients. The size of the lesion is an important factor to observe since it can determine the likelihood of complications. In addition, lesion development is more comfortable to analyse with detailed lesion size data.

PDL laser therapy is the best therapy for superficial IH, although this therapeutic modality cannot inhibit the growth of more profound components. ${ }^{9,16,17}$ The most widely used IH therapy with laser is consistent about its function to decrease the proliferative phase and accelerate rates of involution and resolution. Another therapy for $\mathrm{IH}$ is systemic corticosteroids. This therapy is useful in the IH proliferation phase. Topical corticosteroids can also be used for small and superficial $\mathrm{IH} .{ }^{13,18} \mathrm{IH}$ therapy shall be decided by considering several factors such as location and size of the lesion, psychosocial implications, risks, and therapeutic benefits. ${ }^{17}$ However, the result of the therapy cannot be clearly described on this study.

In conclusion, based on the results of $84 \mathrm{IH}$ patients; most patients were aged less than one year old; mostly females and most patients present with no IH family history. The IH patients mostly presented with a chief complaint of bumps in the facial region with onset of complaints for less than one year, and macular lesions were the most common clinical feature. The most commonly used therapy was laser modality. Other therapy options are propranolol, topical corticosteroids, and antibiotics. The choice of therapy depended on the clinical features and the condition of the IH patient. In addition, periodic monitoring of the IH progression is crucial, given that the development of lesions can vary between individuals.

\section{References}

1. Mathes EF, Frieden IJ. Vascular tumors. In: Goldsmith LA, Katz SI, Gilchrest BA, Paller AS, Leffel DJ, Wolff K, editors. Fitzpatrick's dermatology in general medicine. 8th ed. New York: McGraw Hill; 2012. p. 1456-65.

2. George A, Mani V, Noufal A. Update on the classification of hemangioma. J Oral Maxillofac Pathol 2014; 18(1): 117-20.

3. Léauté-Labrèze C, Harper JI, Hoeger PH. Infantile haemangioma. Lancet 2017; 390(10089): 85-94.

4. Mendiratta V, Jabeen M. Infantile hemangioma: an update. Indian J Dermatol Venereol 2010; 76(5): 469-76.

5. Greene AK. Management of hemangiomas and other vascular tumors. Clin Plast Surg 2011; 38(1): 45-63.

6. Reimer A, Fliesser M, Hoeger PH. Anatomical patterns of infantile hemangioma $(\mathrm{IH})$ of the extremities (IHE). J Am Acad Dermatol 2016; 75(3): 556-63.

7. Callahan $\mathrm{AB}$, Yoon MK. Infantile hemangiomas: a review. Saudi J Ophthalmol 2014; 26(3): 28391. 
8. Restrepo R, Palani R, Cervantes LF, Duarte A, Amjad I, Altman NR. Hemangiomas revisited: the useful, the unusual and the new. Pediatr Radiol 2011; 41(7):895-915.

9. Chinnadurai S, Sathe NA, Surawicz T. Laser treatment of infantile hemangioma: a systematic review. Lasers Surg Med 2016; 48(3): 221-33.

10. Richter GT, Friedman AB. Hemangiomas and vascular malformations: current theory and management. Int J Pediatr 2012; 2012: 645-78.

11. Chen TS, Eichenfield LF, Friedlander SF. Infantile hemangiomas: an update on pathogenesis and therapy. Pediatrics 2013; 131(1): 99-108.

12. Laken PA, Forsythe PL. Infantile hemangiomas: pathogenesis and review of propranolol use. Adv Neonatal Care 2016; 16(2): 135-42.

13. Püttgen KB. Diagnosis and management of infantile hemangiomas. Pediatr Clin North Am 2014; 61(2): 383-402.
14. Moyakine AV, Herwegen B, van der Vleuten CJM. Use of the hemangioma severity scale to facilitate treatment decisions for infantile hemangiomas. J Am Acad Dermatol 2017; 77(5): 868-73.

15. Suh K-Y, Frieden IJ. Infantile hemangiomas with minimal or arrested growth. Arch Dermatol 2010; 146(9): 971-6.

16. Brauer JA, Geronemus RG. Laser treatment in the management of infantile hemangiomas and capillary vascular malformations. Tech Vasc Interv Radiol 2013; 16(1): 51-4.

17. Ames JA, Sykes JM. Current trends in medical management of infantile hemangioma. Curr Opin Otolaryngol Head Neck Surg 2015; 23(4): 28691.

18. Darrow DH, Greene AK, Mancini AJ, Nopper AJ. Diagnosis and management of infantile hemangioma. Pediatrics 2015; 136(4): e1060104. 